\title{
LA NATURALEZA DE LA LÓGICA Y SU CONEXIÓN CON LA ONTOLOGÍA EN ALBERTO MAGNO
}

MAURICIO BEUCHOT

INSTITUTO DE INVESTIGACIONES FILOSÓFICAS

Universidad Nacional autónoma de México

\section{Introducción}

En la filosofía de la lógica desarrollada por los escolásticos medievales, el primer tema era el de la naturaleza misma de la lógica. $\mathrm{El}$ conocimiento de esto se lograba atendiendo i) al objeto de estudio que tiene esta disciplina, ii) al modo -o método- según el cual se estudiaba dicho objeto en la construcción o estructuración de este saber, iii) a la distinción de partes en esa estructurá, es decir, a los diferentes tratados de la lógica, y $i v$ ) a la necesidad, conveniencia y utilidad que competen a la lógica en el campo del saber. Todos esos temas se hallan competentemente tratados en la obra de Alberto Magno (1206-1280), en la que nos basaremos para estudiarlos.

En el presente trabajo intentaremos mostrar dos cosas: a) que estas disquisiciones de los lógicos escolásticos sobre la filosofía de la lógica tenían un fuerte fundamento en la ontología, y $b$ ) que hoy en día, cuando ya abordamos esos fundamentos ontológicos de la lógica explícitamente, tienen mucho que decirnos, sobre todo en la cuestión del tipo de ente que se estudia en la lógica y en la cuestión de la intencionalidad - como la siguió llamando Husserl- con que la mente tiene que aprehenderlo en el aspecto epistémico.

\section{El dominio de la lógica}

Para San Alberto Magno, como para los demás filósofos escolásticos, una ciencia o hábito cognoscitivo se especifica y define por su objeto. El tener objeto es la primera de sus propiedades; las demás propiedades de esa ciencia surgirán de la primera y fundamental. A través de la consideración de su objeto, se puede obtener su esencia o naturaleza, a partir de la cual es posible apreciar mejor sus propiedades. Para estudiar la esencia, lo que procede es establecer la 
definición y la división de la lógica. Y tanto la una como la otra surgen al considerar su objeto.

Acerca del objeto de la lógica, San Alberto nos dice: "Las ciencias lógicas no consideran al ente ni parte alguna del ente, sino las intenciones segundas acerca de las cosas puestas por el discurso". ${ }^{1}$ Pues bien, la metafísica es la que asigna y esclarece los objetos que pertenecen a las demás ciencias. Por. eso Alberto nos da en su Metafísica esta descripción del objeto de la lógica. Al decir que la lógica trata de las intenciones segundas, está diciendo que su objeto es el ente de razón, es decir, no el ente real, sino el ente pensado, y es además un tipo de ente que no se capta con la intención primera del intelecto, o intención directa - con la que captamos el ente real-, síno con la intención segunda o refleja, de la mente - con la que captamos lo relativo a los conceptos mismos. El dominio, pues, de la lógica es el de las propiedades que pertenecen a los conceptos, o a las cosas en cuanto están en el intelecto, los conceptos de segundo orden (o conceptos de conceptos). Es lo que se quiere decir al expresar que trata del ente de razón de segunda intención. Expliquemọs esto.

\section{Ente de razón}

Para entender mejor el ente de razón, al que alude San Alberto, conviene contraponerlo al ente real. El ente real es el que existe en el orden natural de las cosas, fuera de la mente. Entes reales son todos los que conocemos por sensación e intelección dirigidas hacia el exterior, como esta mesa, el cuerpo humano, el alma humana y Dios. Conocemos su's respectivas propiedades, y estas propiedades son reales, porque pertenecen a la cosa en cuanto exterior a la mente. En cambio, el ente de razón corresponde a la cosa en cuanto conocida, y las propiedades que le asignamos en cuanto conocida no son reales, sino mentales. Se trata de los entes de razón con fundamento en la realidad. De este modo, las cosas que son opuestas, que tienen prioridad y posterioridad, que tienen simultaneidad, están en la naturaleza de las cosas. Pero las formas o intenciones de oposición, de prioridad, de posterioridad, de simultaneidad, son puestas por la razón. ${ }^{2}$ Las primeras son entes reales, las segundas son entes de razón. Esto puede verse por el siguiente ejemplo:

1 S. Alberto Magno, In I Metaphysicorum, tr. 1, c. 1; en Opera, ed. A. Borgnet, Parfs, L. Vivès, 1890, vol. 6, p. 3B. Cfr. M. Beuchot, "El espíritu y la obra de San Alberto Magno", en Cuadernos Dominicanos, n. 3 (1978); idem, "Estructura y función de la metafísica según Alberto Magno", en Libro Anual del ISEE, 8 (1980), pp. 213-239; H. Craemer, Alberto Magno, Barcelona, Herder, 1985.

2 S. Alberto, De praedicamentis, tr. 7, c. 1; Borgnet, vol. 1, p. 273A. 
'En efecto, la cosa conocida, por ejemplo 'hombre', considerada como cosa, se atribuye a las cosas reales singulares. Afirmamos con verdad que 'Sócrates es hombre'. Consideradas como conocidas, no tienen sentido tales afirmaciones. Decimos con verdad que 'la idea de hombre es predicable', pero no tiene sentido afirmar que 'Sócrates es predicable'" 3

El ente de razón se divide en negación y relación. Y, más que la negación o privación (como la invidencia, o la ceguera, etc.), es la relación de razón (como la que se da entre definiens y definitum, entre sujeto y predicado, entre antecedente y consecuente, etc.) la que se toma en cuenta aquí como el ente de razón que pertenece a la lógica, del cual decimos que además es de segunda intención cognoscitiva, como trataremos de hacerlo ver a continuación, según los principios escolásticos que manejaba San Alberto.

\section{Intención segunda}

Dice, pues, San Alberto, que el objeto de la lógica es el ente de razón (es decir, la relación de razón) de segunda intención. Para entender el ser de segunda intención, conviene contrastarlo con el de primera intención. El ser intencional corresponde al ser en cuanto conocido, es el existir en el intelecto (es decir, mental o psíquicamente), es otro nombre que se puede dar a la cosa en cuanto está en el intelecto. $Y$ es que el intelecto es de suyo intencional. Se hace la realidad que conoce, $y$ el ente intencional es entonces la cosa conocida en el concepto. Es el concepto mismo en cuanto encierra a la cosa. Desde Brentano y Husserl, pasando por toda la fenomenología, la intencionalidad ha cobrado una gran importancia en la actualidad. ${ }^{4}$

En filosofía, se entiende la intencionalidad en sentido muy amplio, como cierta ordenación, tendencia o proyección a algo. Tomada en este sentido amplio, la intención nọ puede restringirse - como se hace en el lenguaje ordinario - únicamente a la voluntad, como si solamente la voluntad tuviera esa tendencia intencional. El intelecto también tiene su intención, su intencionalidad, está ordenado o polarizado a algo. Ese algo al que está ordenado es la cosa cognoscible y, en cuanto conoce la cosa, el intelecto se hace ella misma, o si se quiere, convierte a esa cosa en cosa-conocida. Y aun puede reflexionar el intelecto y conocer lo que conoce, bajo otra dimensión, a otro nivel (en un segundo orden). Por ejemplo, en una intención

3 E. Chávarri, "El orden de los escritos lógicos de Aristóteles según San Alberto Magno", en Estudios Filosóficos, 9 (1960), p. 105.

1 Cfr. E. Forment Giralt, Fenomenología descriptiva del lenguaje, Barcelona, Publicaciones Universitarias, 1984 (2a. ed.), pp. 181 ss. 
directa puede conocer un hombre sabio y decir "este hombre es sabio"; pero también puede conocer lo que ha conocido y decir que en lo que dijo hay adjetivo demostrativo, sustantivo, verbo y adjetivo calificativo; o que hay sujeto y predicado. Hay, pues, dos niveles de intencionalidad, dos intenciones. Por eso se habla de intención primera e intención segunda. Con ellas la cosa pasa a tener en el intelecto un ser intencional, y el ser intencional es, así, una forma vicaria, que hace las veces de y representa a la cosa en la inteligencia; pero como el ser intencional es vicario, con carácter de vehículo, tiene una existencia efímera, los escolásticos decían: "pordioseada" - "mendigada" (emendicata) al ser real. Es un ser que sólo se da en la mente.

La primera intención de la mente versa sobre la cosa real de manera directa; la segunda intención de la mente versa sobre nuestro mismo conocimiento de la cosa, de modo reflejo. En la primera nos apropiamos cognoscitivamente de la realidad y la convertimos en racional, la transformamos en ser de razón, en concepto (directo). En la segunda, reflexionando sobre nuestros conceptos, los organizamos y disponemos en orden a la predicación; como cuando los dividimos en sujeto, predicado y término medio de un silogismo, antecedente y consecuente, o premisas y conclusión. No hablamos ya de las cosas, sino de las cosas en cuanto conocidas, esto es, de nuestros conceptos, mediante otros nuevos conceptos de nivel u orden superior. Por eso también se llama "concepto directo" al de primera intención, y "concepto reflejo" al de segunda intención. (El llamarlo "de segunda intención" ya nos indica que es de orden superior al de primera, como un metaconcepto de otro.)

El ente de razón de primera intención es la misma cosa real que ha pasado de un modo de ser real a un modo de ser racional o mental. Se ha abstraído su esencia, se ha convertido en universal. El ente de razón de segunda intención es el modo de ser lógico que asignamos a los universales o conceptos, según cierta ordenación que la mente introduce. Aunque están fundados en la realidad, de la que surgieron por abstracción, por comparación de unos con otros, establecemos su ordenación dentro de las operaciones lógicas.

Como se trata de una ordenación que introduce la comparación intelectual o racional de conceptos, la segunda intención pertenece en propiedad a la relación racional, la cual, junto con la negación (o privación), constituye los entes de razón. Por eso a esas relaciones que son entes de razón se les llama relaciones de razón de segunda intención. Relaciones de este tipo son las que se dan entre sujeto y predicado (es decir, relación de predicabilidad o predicación), o entre antecedente y consecuente (es decir, relación de consecuencia 
o ilación inferencial). Y este tipo de entes de razón, que son las relaciones de razón de segunda intención, o intenciones segundas, son las que constituyen según San Alberto el objeto de la lógica.

\section{El objeto de la lógica}

En una ciencia, el objeto - para San Alberto Magno-- puede ser de diversos tipos. Dejando de lado otras divisiones, podemos dividir el objeto de una ciencia en objeto material y objeto formal. El objeto material es el objeto en su máxima extensión y amplitud, al cual conviene diferenciar. El objeto formal es lo que da esa diferenciación o especificación al anterior; este nuevo objeto es, a su vez, doble: i) Objeto formal terminativo $u$ objeto formal quod -como se le llamaría después, es decir, el objeto que se considera-, el cual es propiamente aquello que la ciencia estudia, lo que específicamente considera dentro del objeto material, y que es además aquello que vertebra o estructura a todo el tratamiento o estudio. $Y$ ii) objeto. formal motivo u objeto formal quo - es decir, por el cual-, que es la luz intelectiva o el tipo de abstracción intelectual por el cual se capta y se estudia el objeto propio de la ciencia.

De acuerdo con ello, veamos cuál es con toda precisión el objeto de la lógica - en Alberto Magno. Grosso modo hemos dicho que a éste lo constituyen las relaciones de razón de segunda intención. Veamos con más detalle cómo es esto. En primer lugar, el objeto material o amplio de la lógica es la relación de inferencia, consecuencia o argumentación; en efecto, la lógica pretende establecer las reglas para pasar de lo conocido a lo ignorado. "Y en esto consiste la argumentación, en cuanto que la argumentación es el raciocinio con el que la mente arguye y convence por la relación de lo conocido a lo ignorado acerca de la ciencia de lo ignorado. $Y$ entre las especies de la argumentación, la principal es el silogismo." 5 Esto es, todos los demás elementos que se tratan en la lógica son considerados en cuanto ordenados a la argumentación. Asimismo, el objeto formal terminativo (o quod) son las relaciones de razón de segunda intención, porque todos los elementos que se estudian en la lógica se pueden ver y estructurar como relaciones de este tipo. $Y$ su objeto formal motivo (o quo) es la abstracción formal de tercer grado -es decir, la abstracción de grado más alto, igual en grado que la metafísica-, pero distinta de la metafísica, pues esta última alcanza ese mismo grado en el orden intelectivo de la primera intención, mientras que la lógica lo alcanza - como hemos visto- en el orden de la segunda intención intelectual.

5 S. Alberto, De praedicabilibus, tr. 1, c. 4; Borgnet, vol. 1, p. 6B. 
Definición de la lógica

Según los principios de Alberto Magno, se podría, pues, establecer la siguiente definición de la lógica: es la ciencia y arte que estudia las operaciones de la mente estableciendo orden en las relaciones de razón de segunda intención que ésta elabora, a la luz de la abstracción formal de tercer grado en el plano de las segundas intenciones.

Hemos añadido en esta definición que la lógica es a la vez ciencia y arte (o técnica). Esto no es incompatible. Es ciencia porque investiga su objeto, elabora sus definiciones y principios, y demuestra las propiedades que asigna a su dicho objeto. $Y$ es arte en cuanto aporta reglas para dirigir las operaciones de la mente y establecer bien las relaciones de razón que ellas operan. Así, la lógica es también un instrumento, el órganon para la adquisición de las otras ciencias. Además, hay una lógica natural, pero es imperfecta; y por eso es necesaria la lógica técnica (o como arte: techne), ya que lo natural ès perfeccionado por el arte. De ahí que la lógica sea tan necesaria y útil.

\section{División de la lógica}

Como es sabido, toda definición se efectúa con arreglo a algún criterio. Si esto es así, el mejor criterio para efectuar la división de la lógica sería - en el sistema albertino- su propia definición. Tomemos, pues, como fundamentum divisionis la misma definición de la lógica. "De este modo la lógica contiene tantas partes cuantas relaciones de razón de segunda intención. San Alberto, si bien no siempre explícitamente, reduce esas relaciones a tres, y conforme a ellas ordena las obras lógicas del Estagirita. Así, para exponer la relación de predicabilidad 'en cuanto está en los elementos ordenados', escribió las Categorías, que constituye la primera parte de su lógica. A la relación de predicabilidad sigue connaturalmente la relación de predicación, que se analiza ampliamente en la obra De la interpretación: segunda parte de la lógica. El tratado más extenso de la relación de ilación, tercera parte del Órgano, se expone en los restantes libros: Analíticos primeros, Analíticos segundos, Tópicos, Elencos sofísticos, Retórica y Poética." 6

Llamará la atención que los escolăsticos a veces incluyen la retórica y la poética en el ámbito de la lógica. Pero es que las entendían como disciplinas que eran por lo menos ampliaciones del tratado 
de la argumentación. En efecto, la retórica estudiaba la argumentación persuasiva, a la que le bastaba alcanzar lo verosímil, si no podía llegar a la verdad. Y la poética estudiaba la argumentación fantasiosa, a la que no se le exigía llegar ni siquiera a lo verosímil en sentido fuerte, y mucho menos a la verdad, pero que - tocándose con lo verosímil - tenía que guardar una coherencia indispensable en el mismo ámbito de lo metafórico, lo fantástico, lo poético. Y, por otra parte, resulta fácil comprender y aceptar que los Tópicos tienen la lógica de lo probable o del argumento plausible, mientras que los Analíticos tienen la lógica apodíctica o del argumento necesario, al tiempo que los Elencos contienen la argumentación falaz. De esta forma, todos los elementos de la lógica se ordenan en definitiva a construir la buena argumentación.

$Y$ también resulta claro que las partes en las que San Alberto divide la lógica, en seguimienta de Aristóteles, son las mismas que resultan de dividirla según las tres operaciones del intelecto: simple aprehensión, composición-división y raciocinio. En concordancia con eso, Alberto señala tres tratados: a) el de la predicabilidad, b) el de la predicación y $c$ ) el de la consecuencia, inferencia o argumentación. Veamos en qué consiste cada úno de ellos, para completar nuestra idea del objeto de la lógica.

\section{La predicabilidad}

La consecuencia o argumento consta de proposiciones, y aun puede vèrse ella misma como un tipo de proposición: la proposición mediata (es decir, la que tiene un término medio silogístico, explicitando el cual puede contruirse un silogismo in forma); por ello la teoría de la consecuencia lógica se basa en la predicación; y, de acuerdo con eso, conviene preparar la ordenación inferencial estableciendo los elementos y la razón de ese orden. Hay que estudiar la predicación para poder estudiar la inferencia. Y el orden de la relación de predicación se prepara disponiendo la relación de predicabilidad de los elementos que van a constituirla. Esto es, los elementos que se pueden predicar, que pueden ser los predicados en la predicación. Surge así un doble problema: el de los modos como se pueden atribuir los predicados (es decir, el tratado de los predicables) y el de los tipos concretos de predicado que hay (es decir, el tratado de los predicamentos o categorías).

Para que un término se pueda predicar de otro es requisito que tenga mayor o, por lo menos, igual universalidad. Por eso el fundamento de la predicabilidad es la universalidad. ${ }^{7}$ En consecuencia,

7 Cfr. S. Alberto, De praedicabilibus, tr. 2, c. 1; Borgnet, vol. 1, p. 17A. 
el tratado de la predicabilidad es el tratado de los distintos universales y sus correlaciones. Alberto encuentra la razón de orden de la predicabilidad en la Isagoge o Introducción de Porfirio al libro de las Categorías de Aristóteles. El orden mismo de la predicabilidad lo encuentra en las Categorías (que considera obra auténticamente aristotélica), y, como advierte que es un tratado muy breve, encuentra que puede ser complementado con el tratado De sex principiis de Gilberto de la Porrée. Con base en ellos desarrolla su estudio de la predicabilidad.

La primera operación de la mente es el conocimiento de lo simple o incomplejo como elemento de predicación, y se basa en lo universal, que contiene la relación de predicabilidad. San Alberto da primeramente una teoría del universal. ${ }^{8}$ El universal no tiene propiamente definición, pero se puede definir impropiamente como "lo que, estando en uno, es de suyo apto para estar en muchos". 9 No tiene definición propia porque el universal es algo analógico, no unívoco - que es lo que propiamente tiene definición.

Para saber lo que es un análogo, hay que distinguirlo de lo unívoco y lo equívoco. Univoco es un término que designa varios individuos, o se predica de varios sujetos, con la misma significación. Por ejemplo, "hombre" designa a, y se predica de, Sócrates, Pedro, etc., de igual modo y con el mismo significado. Equívoco es el término que designa a varios individuos, o se predica de varios sujetos, con diferente significación. Por ejemplo, "gato" designa y se predica de diferente modo y en diverso sentido del animal y del instrumento, etc. $\mathrm{Y}$ análogo es el término que designa y se predica de suyo con diferente significación, pero, bajo algún respecto, con la misma. Por ejemplo, "viviente" designa de suyo algó distinto, y bajo cierto aspecto idéntico, aplicado al vegetal, al animal y al hombre.

El universal es análogo porque no se dice que todos los universales sean igualmente universales o igualmente predicables. ${ }^{10}$ Hay más y menos en ellos (es decir, algo per prius y algo per posterius). Por eso el universal no tiene definición propia, unívoca. Tiene que buscársele una definición impropia y aproximativa, como "lo que puede predicarse de muchos" ${ }^{11} \mathrm{Y}$ la diversidad de predicabilidad da origen a distintos modos de predicables.

8 Cfr. M. Beuchot, El problema de los universales, México, Facultad de Filosofía y Letras de la UNAM, 1981, pp. 159-195, que es el capítulo dedicado a San Alberto Magno.

9 S. Alberto, De praedicabilibus, tr. 2, c. 1; Borgnet, vol. 1, p. $17 \mathrm{~B}$.

10 Cfr. ibid., tr. 2, c. 9; Borgnet, vol. 1, p. 39A y tr. 8, c. 1; Borgnet, vol. 1, p. $125 \mathrm{AB}$.

Pi Ibid., tr. 2, c. 1; Borgnet, vol. 1, p. $17 \mathrm{~B}$. 
Los predicables son cinco: género, especie, diferencia específica, propiedad y accidente. El género representa el aspecto determinable de la esencia; la diferencia, el aspecto determinante de la misma; la especie representa a la especie ya determinada; la propiedad, una característica que acompaña a la esencia de manera necesaria, aunque no esencial; y el accidente, una característica que acompaña a la esencia de manera completamente contingente, y no le afecta en nada a esa esencia el que se le quite o se le añada. Ejemplificando esto con "hombre", podemos decir que el género es "animal", su diferencia especifica es "racional", su especie es "animal racional", su propiedad es "risible" y su accidente es "rubio". Son los cinco modos como se puede predicar un atributo.

Los cinco predicables enlistados son suficientes en número, y no hace falta añadir ni quitar ninguno; porque el universal, en cuanto predicable y que inhiere en muchos, o inhiere como esencia o como accidente. "Si inhiere como esencia, o inhiere como toda la esencia o como parte esencial. Si inhiere como toda la esencia formal, consta que es la especie, porque la especie constituye como un todo formal el ser de los individuos, de los que se predica, ya que todo lo que se da después de la especie, pertenece a la materia o a los principios individuantes. Si como parte esencial, entonces o según la potencia en la que está el ser por incoación, o es parté según el acto en que está el ser ya acabado. Del primer modo es el género, y del segundo es la diferencia. Pero si inhiere como accidente, o inhiere como accidente de la naturaleza, que es causado por la misma aptitud de la naturaleza y emana de ella, o como accidente común, el cual es accidente del individuo. Del primer modo es el propio y del segundo es el accidente." 12

Lo que se puede ordenar en géneros, especies, diferencias, propiedades y accidentes son los predicamentos. Los predicamentos surgen de la ordenación de los predicables. $Y$ es que la primera operación de la mente consiste en ordenar lo que se puede componer con otro. Ahora bien, la primera ordenación versa sobre lo que se puede predicar, y esto trata de la conexión o composición de lo universal con lo particular, pues lo más universal es lo que se puede predicar de lo menos universal o más particular. Y esto perteneció a los predicables, donde se tomaba el orden desde el predicado hacia el sujeto. Ahora se pasa a estudiar los elementos integradores en cuanto pueden ser sujetos o predicados, y en esto consiste la disposición de los diez géneros supremos o predicamentos, ${ }^{13}$ que se

Ibid., tr. 2, x. 1; Borgnet, vol. 1, pp. 17B-18A; cfr. ibid., tr. 2, c. 9; Borgnet, vol. 1 , p. $40 \mathrm{AB}$.

13 Cfr. idem, De praedicamentis, tr. 1, c. 1; Borgnet, vol. 1, p. $149 \mathrm{~B}$. 
pueden disponer en los predicables. $O$, más correctamente, que se pueden ordenar según cada uno de los predicables.

Los predicamentos, pues, son lo que se ordena bajo cada uno de los predicables siguiendo una jerarquía de más a menos universal, desde un género supremo (o categoría) hasta los individuos. En esta línea de los predicamentos, todo lo que cae bajo cada uno de ellos tiene univocidad. Son en número de diez: la substancia y los nueve accidentes, a saber, cantidad, cualidad, relación, acción, pasión, tiempo, lugar, situación y posesión (o hábito). Tanto los predicables como los predicamentos, por ser los constitutivos o elementos de la predicación, son algo simple o incomplejo, y la predicación algo complejo, en lo que se unen (afirmando) o se desunen (negando).

\section{La predicación}

La predicación es el segundo acto de la mente y, por trabajar con los conceptos o términos como elementos, es algo complejo: compone y divide (une o desune, por la afirmación y la negación, respectivamente). La predicación se muestra en forma de interpretación u oración, que tiene como clase principal la enunciación o proposición. En efecto, la interpretación difiere de la enunciación como el todo difiere de la parte: "La interpretación se hace de muchos modos. La enunciación no expresa sino que una cosa se dice o se predica de otra. Y, aunque la enunciación sea el principal tipo de interpretación, sin embargo, no equivale a la interpretación, que expresa la exposición de la cosa de todos los modos, ya en parte, como el nombre y el verbo, ya en su totalidad, como en cuanto a la cualidad de afirmativa o negativa, o como inhiriendo universal o particularmente, o de manera finita o infinita, o en una oración o en varias: de las cuales nada dice en su nombre la enunciación. Y la interpretación, según lo ideal, no se puede realizar sino haciéndose de todos los modos que se han dicho". ${ }^{14}$

Es decir, la interpretación u oración tiene muchos modos: aseverativo, interrogativo, deprecativo, imperativo, potencial, etc. Pero, de entre ellos, sólo es enunciado o proposición la oración que va en modo indicativo o asèverativo, pues es el único que cumple perfectamente con la afirmación y la negación, y por ello el solo que puede tener verdad o falsedad. La enunciación representa perfectamente

14 Idem, In I Peri hermeneias, tr. 1, c. 1; Borgnet, vol. 1, p. 374A. Cfr. M. Beuchot, La filosofía del lenguaje en la Edad Media, México, Instituto de Investigaciones Filosoficas de la UNAM, 1982, pp. 65-82 (el capitulo dedicado a Alberto Magno). 
la felación de predicación, pues en ella es donde se ve con mayor claridad que algo se predica de algo. ${ }^{15}$

En los nombres "oración", “interpretación”, "enunciación” y "proposición" podemos distinguir matices diversos: La oración es la representación de lo real a través de la articulación de la voz significativa. La interpretación es la oración que dispone en palabras lo que hay en la realidad, intentando expresarla. La enunciación es una clase de la interpretación, y representa la relación de predicación, pues en ella algo se predica de algo. La proposición o sentencia es una clase de la enunciación, pues es la misma enunciación en cuanto parte de una consecuencia, especialmente del silogismo. (Por eso ella debe estudiarse en el tratado de la consecuencia, como materia del silogismo.) Lo que propiamente pertenece al tratado de la predicación es la interpretación, la relación de las oraciones con los significados (volitivos y cognoscitivos), esto es, la oración y la interpretación, especialmente la enunciación, pues es el tipo de interpretación que representa perfectamente la relación de predicación.

La enunciación o enunciado se divide en simple y compuesta (o categórica e hipotética), afirmativa y negativa, universal y particular. La propiedad principal es la oposición, que dimana de la división en afirmativa y negativa, añadida la otra división en universal y particular. $Y$ todo ello se toma en cuenta para examinar sus comportamientos en la consecuencia o inferencia argumentativa.

\section{La consecuencia}

La tercera y última operación de la mente es el raciocinio, por el cual pasamos de proposiciones conocidas (premisas o antecedentes) a proposiciones nuevas (conclusiones o consecuentes). El raciocinio, argumentación o discurso representa la relación de ilación, de inferencia o de consecuencia. Hay muchos modos de consecuencia, pero la principal es la silogística, y, entre los silogismos, el más perfecto es el categórico. (San Alberto lo contrapone al hipotético, en el sentido de que el hipotético no confiere un conocimiento cierto, mientras que el categórico sí lo da.) ${ }^{16}$

La consecuencia se puede estudiar de dos maneras: o en cuanto a la razón de consecuencia, esto es, en cuanto a la sola forma del raciocinio, por la cual vemos que el consecuente se sigue del antecedente,

Borgnet, vol. 1, p. 407B.

16 Cfr. idem., In I Posteriorum Analyticorum, tr. 2, c. 1; Borgnet, vol. 2, p. $21 \mathrm{~B}$ y tr. 3, c. 8; Borgnet, vol. 2, pp. 88A ss. 
y entonces examinamos su validez; o en cuanto a la razón del consecuente, esto es, en cuanto a una de las proposiciones que constituyen su materia, y entonces examinamos su verdad, que puede ser de cuatro tipos: necesaria, imposible y contingente, posible o probable, y esta última puede ser, a su vez, auténticamente probable o aparentemente probable. Cuando la proposición consecuente es de materia necesaria, se origina el silogismo demostrativo; cuando es de materia imposible, se origina el silogismo falsígrafo; cuando es de materia auténticamente probable, se origina el silogismo dialéctico; y cuando es de materia aparentemente probable, se origina el silogismo litigioso o sofístico. $Y$ así tenemos todas las clases de silogismo, según las cuales se ordenan los tratados de la inferencia: analítica, tópica (incluyendo retórica y poética) y sofística. Tenemos, con ello, redondeadas todas las partes de la lógica en su culminación, que es la teoría de la argumentación.

\section{Conclusión: actualidad de esta doctrina}

Es muy clara la relación de esta filosofía de la lógica, desarrollada por los escolásticos, con la ontología que adoptaban. En el caso de San Alberto, se trata de una ontología aristotélica; en otros casos era una ontología de signo platónico o aun de signo nominalista.

Y también, como resultado de nuestro recorrido, podemos apreciar que estos estudios nos enseñan mucho en la actualidad, cuando la reciente filosofía de. la lógica - Quine, Susan Haack, Hintikka y otros- busca la raigambre ontológica de las nociones lógicas que empleamos. El esfuerzo escolástico nos enseña que tenemos que tratar de precisar el status ontológico del objeto de la lógica, que hemos de intentar buscar si tiene o no correspondencia con lo real y, si la tiene, de qué tipo es. También nos manifiesta la importancia de las consideraciones gnoseológicas o epistémicas en la filosofía de la lógica - que por supuesto no entran en la lógica misma, pero sí en la filosofía de la lógica, y a veces se han evitado so pretexto de excluir psicologismos-: cómo se conoce el ente lógico, qué intencionalidad cognoscitiva requiere por parte de la mente y cómo esa intencionalidad se despliega en predicable, predicativa (o judicativa) e inferencial. $Y$ se tiene que examinar cómo todo esto se encuentra virtualmente contenido en el objeto que se asigne a la lógica, lo cual exige tener un gran cuidado al establecerlo y precisarlo. Mutatis mutandis, en estas reflexiones y especulaciones medievales que hemos señalado -y que se encuentran dentro de la filosofía de la lógica-, los estudiosos modernos encuentran puntos de interés común, indicadores y pistas con los que pueden incrementar el propio desarrollo de la actual filosofía de la lógica. 